\title{
Analytical treatment of small scales matter power spectrum in coupled scalar field (CSF) cosmology
}

\author{
Fargiza A. M. Mulki ${ }^{1, *}$, and Hesti Wulandari ${ }^{1}$ \\ ${ }^{1}$ Astronomy Research Division, Faculty of Mathematics and Natural Sciences, Institut Teknologi \\ Bandung, Jl. Ganesha 10, Bandung 40132, Indonesia
}

\begin{abstract}
In this paper, we reconstruct matter power spectrum of a cosmological model in which coupled scalar field acts as dark energy. The coupled scalar field (CSF) dark energy we propose here is a generalized model of quintessence, $\mathrm{k}$-essence or phantom coupled to dark matter (nonbaryonic matter) via a coupling constant. The existence of coupling between dark energy and matter allows energy to transfer between them, which may give rise to different observational signatures, especially at perturbation level, including matter power spectrum. In this work, through analytical exploration we studied that possible signature in matter power spectrum that maybe induced by this mode.
\end{abstract}

\section{Introduction}

The standard model of cosmology (the $\Lambda$ CDM model), is constructed by two dark components of the universe, namely the cosmological constant and cold dark matter. Recent results of Planck CMB mission are in very good agreements with base $\Lambda$ CDM model [1]. Despite being successful in explaining a large set of cosmological observations, the $\Lambda$ CDM model suffers from several problems. One of the problems is concerning the interpretation of the cosmological constant. Designating the cosmological constant as vacuum energy stored in spacetime leading to severe fine tuning problem [2]. This serious problem has evoked persistent interests in extending cosmology beyond the standard model, including proposals of dynamically changing scalar field as alternative models of dark energy, such as quintessence, k-essence, and phantom [3 - 10]. Considering that Planck CMB results allow dark energy to enter phantom domain $\left(\omega_{D E}<-1\right)$, and the fact that the energy density value of dark energy today is similar in order of magnitude with that of matter [11], generalizations of ordinary scalar field into phantom dark energy and uncoupled scalar field into coupled scalar field have been suggested [6,12-18].

One way to yield phantom scalar field is by allowing scalar field to have negative kinetic energy, so that its state parameter $\omega_{\phi}$ would exceed the lowest value of ordinary scalar field (see ref. [6]). Different types of couplings have been put forward, one type of coupling that has been put forward is coupling between matter and dark energy via a coupling constant $Q$

\footnotetext{
* Corresponding author: fargiza@as.itb.ac.id
} 
$[12,13,15,16]$. Several authors have explored the effect of such a coupling to the perturbation dynamics of universe $[3,4,13,15,16]$. In this work, we reconstructed matter power spectrum following CSF-DE and investigated how coupling values can affect the shape of matter power spectrum. Whereas, the inquiry of differences between phantom and ordinary field of CSF-DE has been performed by analyzing CMB model generated by using CAMB codes for various $\omega_{D E}$ [19]. ]. In this work, we have reconstructed matter power spectrum following CSF-DE and investigated how coupling values can affect the shape of the matter power spectrum.

\section{The Dynamics of Coupled Scalar Field (CSF-DE)}

The model used in this work is in the form of lagrangian:

$$
L(X, \phi)=X-V(\phi)-Q \rho_{m} \phi
$$

where $Q, \rho_{m}$ and $\phi$ stand for coupling constant, general matter density and scalar field respectively, and $X=-\frac{1}{2} g^{\mu \nu} \nabla_{\mu} \phi \nabla_{\mu} \phi$ is the kinetic component of scalar field. For lagrangian in eq. (1) we can derive the equation of motion for scalar field in Friedmann universe,

$$
\phi^{\prime \prime}+2 \mathrm{H} \phi^{\prime}+a^{2} V_{, \varphi}=-Q \rho_{m},
$$

which is usually known as Klein-Gordon equation. Scalar field coupled to general matter satisfies the condition

$$
\nabla_{\mu} T_{\nu}^{\mu}= \pm Q T_{M} \nabla_{\nu} \phi
$$

where the plus $(+)$ sign refers to matter and radiation, minus $(-)$ sign refers to scalar field, and $T_{M}$ is the trace of fluid energy-momentum tensor. Complete derivation of the dynamics of the model has been performed by several authors $[6,15,17,18]$, so that here we are only interested in using some results obtained mainly by [15] that are relevant to our objectives. The dynamical equations for scalar field with potential $V=V_{0} e^{-\lambda \phi}$ are given by [15]

$$
\begin{aligned}
& \frac{d x}{d N}=-3 x+\frac{\sqrt{6}}{2 \varepsilon} \lambda V_{0} y^{2}+\frac{3}{2} x\left[\left(1-\omega_{m}\right) \varepsilon x^{2}+\left(1+\omega_{m}\right)\left(1-V_{0} y^{2}\right)\right]-\frac{\sqrt{6}}{2 \varepsilon} Q\left(1-\varepsilon x^{2}-V_{0} y^{2}\right), \\
& \frac{d y}{d N}=-\frac{\sqrt{6}}{2} \lambda x y+\frac{3}{2} y\left[\left(1-\omega_{m}\right) \varepsilon x^{2}+\left(1+\omega_{m}\right)\left(1-V_{0} y^{2}\right)\right],
\end{aligned}
$$

where $\varepsilon=+1$ for ordinary field and $\varepsilon=-1$ for phantom fields. Above equations are autonomous equations which have infinite number of solutions that move together passing critical points $x$ and $y$ which correspond to particular epochs of the universe. Here we mention several domination stages which are relevant to our next discussion, and for practical reason we ignore the radiation dominated era. Matter dominated era corresponds to $x=$ $-\sqrt{6} Q / 3 \varepsilon, \Omega_{\phi}=2 Q^{2} / 3 \varepsilon$, and $\omega_{e f f}=2 Q^{2} / 3 \varepsilon$, matter scaling era corresponds to $Q x=$ $-\sqrt{6} \omega_{e f f} / 2$, and scalar field dominated era corresponds to $x=\lambda / \sqrt{6} \varepsilon, y=\left[1 / V_{0}(1-\right.$ $\left.\left.\lambda^{2} / 6 \varepsilon\right)\right]^{1 / 2}, \Omega_{\phi}=1, \omega_{\phi}=\omega_{e f f}=-1+\lambda^{2} / 3 \varepsilon . x$ and $y$ are dimensionless variables that are introduced to dynamical analysis in phase space, $\Omega_{\phi}$ is density parameter of CSF-DE, $\omega_{e f f}$ is efective equation of state parameter, $\omega_{\phi}$ is equation of state parameter of CSF-DE, $\lambda$ and $V_{0}$ respectively are constant and initial potential (see for details ref. [15]).

To achieve our goal in constructing cosmological power spectra, we need to carry out analysis of perturbation dynamics. In cosmological perturbation theory, structures can be regarded as small fluctuations in the past that evolves with time. For the reason that the 
dominant force driving the universe on large scales is gravity, we give perturbation to gravity in which mathematically can be expressed by expanding Einstein field equation into Taylor series written as follows

$$
\delta\left[R_{\mu \nu}-\frac{1}{2} g_{\mu \nu} R\right]=\delta\left[8 \pi G T_{\mu \nu}\right] .
$$

By evaluating and simplifying eq. (4) together with perturbing eq. (3), we can obtain following equation [15]

$$
\frac{d^{2} \delta_{m}}{d N^{2}}+\left(\frac{1}{2}-\frac{3}{2} \omega_{e f f}+\sqrt{6} Q x\right) \frac{d \delta_{m}}{d N}-\frac{3}{2}\left(1-\Omega_{\phi}\right)\left\{1+2 Q^{2}\left(1-\Omega_{\phi}\right)\right\} \delta_{m}=0
$$

The solutions are in the form $\delta(a)=C_{1} a^{\gamma_{1}}+C_{2} a^{\gamma_{2}}$, with

$$
\gamma_{1,2}=\frac{1}{\varepsilon}\left[\frac{3 Q^{2}}{2}-\frac{\varepsilon}{4} \pm \frac{1}{12} \sqrt{192 Q^{6}-576 \varepsilon Q^{4}+432 \varepsilon^{2} Q^{2}+3324 Q^{4}-252 \varepsilon Q^{2}+225 \varepsilon^{2}}\right],
$$

for matter dominated era, and

$$
\gamma_{1,2}=\frac{1}{2}\left[-5+\frac{3 \lambda^{2}}{2 \varepsilon} \pm\left(5-\frac{3 \lambda^{2}}{2 \varepsilon}\right)\right]
$$

for scalar field dominated era.

Based on the results in eq. (5) and (6) we see that, for ordinary fields, the existence of coupling $Q$ triggers additional growth rate so that structures grow faster than in the cases of uncoupled and $\Lambda \mathrm{CDM}$ models. On the other hand, phantom fields with negative kinetic energy invert the flow of energy current, so that structures grow slower than in the cases of uncoupled and $\Lambda$ CDM models. As for the time in the distant future, both ordinary and phantom fields will break down the existing structures, but with a little distinction between them, i.e. in phantom scenario the decay occurs stronger than as in the ordinary one, indicating an explosive expansion of the background universe or big rip.

\section{Matter Power Spectrum}

In this section we will discuss the possible signatures that may be generated by CSF-DE model. One of the main promising signatures to investigate is matter power spectrum. Here, we will construct matter power spectrum originally starting from CSF-DE model, in which all the aforementioned equations will analytically be transformed into observable parameters.

\subsection{Matter transfer function}

To construct matter power spectrum we need a function named transfer function. Transfer function is a function that map perturbation in the past into its value we observe today. The first step we need to do is connecting perturbation variables over spaces $x$ and scales $k$. Therefore, we need Fourier transform that by definition is expressed as

$$
\delta(\vec{x}, t)=\frac{V}{(2 \pi)^{3}} \int \delta(\vec{k}, t) e^{i(\vec{k} \cdot \vec{x})} d \vec{k},
$$

where $V$ is a volume that large enough to respect cosmological principle, and $\delta(\vec{x}, t)$ is density contrast which satisfies $\rho(\vec{x}, t)=\bar{\rho}(t)(1+\delta(\vec{x}, t))$. Statistical properties of perturbation are determined well by infinite number of correlation functions $\zeta_{n}$ that states how the density of a region correlated with those of other region(s), for example two correlation function $\zeta_{2}=\left\langle\delta\left(x_{1}\right) \delta\left(x_{2}\right)\right\rangle$. 
By decomposing correlation function $\zeta_{2}$ into Fourier space, we obtain

$$
\zeta_{2}(\Delta)=\frac{V}{(2 \pi)^{3}} \int|\delta(\vec{k})|^{2} e^{-i \vec{k} . \Delta} d \vec{k} .
$$

The average values of amplitude in eq. (7) is known as power spectrum, which mathematically is expressed as:

$$
P(k) \equiv\left\langle|\delta(\vec{k})|^{2}\right\rangle .
$$

Next, imagine a universe as an open system which has a set of physical laws and conducts its physical processes that is implicitly contained by a function $T(k, t)$. This function transform input into output. In cosmological context, we may regard all perturbations entered the horizon as inputs, and the perturbation observed today as output. Therefore, we may write

$$
\delta(k, t)=T(k, t) \delta\left(k, t_{0}\right) .
$$

Here we consider two cases: perturbations that enter the horizon during radiation dominated era, and those that enter the horizon during matter dominated era. We do not consider all perturbations that entered at dark energy dominated era because we know that from observations there are not structures found as large as the scales of horizon at dark energy era.

For small scales in which perturbation enter the horizon at radiation dominated era and undergo physical processes generated during radiation era, matter era, and finally coupled scalar field era. Therefore, we may write all these processes mathematically as

which yields

$$
k^{3 / 2} \delta(k, a=1) \approx \underbrace{\delta(k, a)}_{M D} \times \underbrace{\delta(k, a)}_{R D} k^{3 / 2}
$$

$$
\begin{gathered}
T_{S}(k) \approx \frac{2 a_{e q}^{\gamma} \ln \left(a_{e q}\right)}{a_{e q}^{\gamma}-1}\left(\frac{200 \pi \sqrt{\Omega_{R, 0}}}{a_{e q} c k}\right)^{\gamma}, \\
\gamma=\frac{1}{\varepsilon}\left[\frac{3 Q^{2}}{2}-\frac{\varepsilon}{4}+\frac{1}{12} \sqrt{192 Q^{6}-576 \varepsilon Q^{4}+432 \varepsilon^{2} Q^{2}+3324 Q^{4}-252 \varepsilon Q^{2}+225 \varepsilon^{2}}\right] .
\end{gathered}
$$

where we have used eq. (5) (8), and relation:

$$
a=\frac{2 \pi H_{0} \sqrt{\Omega_{R, 0}}}{c k}
$$

for small scales. For large scales in which perturbation enter the horizon at matter dominated era, it experienced the physical processes generated during matter era and coupled scalar field era. However, since we have no evidences for the existence of structures largest than the horizon size of universe at matter-dark energy equality, we can assume that there is no dynamical evolution of perturbation during scalar field dominated era at linear level. Therefore, we can write the process as follows:

$$
k^{3 / 2} \delta(k, a=1) \approx \underbrace{\delta(k, a)}_{M D} k^{3 / 2}
$$

which yields,

$$
T_{L}(k) \approx 1,
$$

means that there is no perturbation change in scalar field domination era in the linear regime. 


\subsection{Power spectrum on small scales}

In many literature, power spectrum is usually expressed as:

$$
P(k)=N_{s} k^{n_{s}} T^{2}(k)
$$

where $N_{s}$ is a normalization factor that can be confirmed with CMB power spectrum, and $n_{s}$ refers to scalar spectral index. $S$ subscription refers to scalar perturbation. Because we focus only on small scales region, $N_{s}$ maybe different from amplitude of primordial power spectrum $A_{S} . N_{S}$ can be obtained by calibrating our calculation to CMB simulation such as CAMB code [19]. From our simulation with CAMB we roughly get $N_{s} \sim 10^{8}$. It is also possible that the value of $n_{s}$ depends on scales, meaning that the observed power spectrum deviates from Harrison-Zeldovich spectrum $\left(n_{s}=1\right)$. To see how $n_{s}$ could be differ from invarint-scale spectrum value, we plot power spectra with various $n_{s}$ for $\mathrm{Q}=0$ in Figure 1 .

Figure 1 shows that our transfer function model for CSF-DE (ordinary scalar fields, $\varepsilon=+1$ ) deviates from invariant-scale spectrum expected by standard inflation theory. For power spectrum of eq. (10) and transfer function of eq. (9), it is well confirmed by this result that $n_{s}=0$ is more preferable than $n_{s}=1$. So we propose a power spectrum in the form of eq. (10) with:

$$
n_{s} \approx \begin{cases}1 & k \ll k_{e q} \\ 0 & k \gg k_{e q}\end{cases}
$$

Eq. (11) appears as a requirement for matching model with data. To acquire appropriate values in the small scale region, we make grids for $k$ values in the range of $0.2<k<2.7$, and calibrate our result for $Q=0$ and $n_{s}=0$ with simulation for standard model ( $\Lambda \mathrm{CDM}$ ) using CAMB code. As the result we obtain $N_{s} \approx 6.5 \times 10^{8}$. In Figure 2 , we show power spectra on small scales for various $Q$ with $n_{s}=0$. We also plot the reference CAMB simulation for appropriate range, and Lyman- $\alpha$ data from [20].

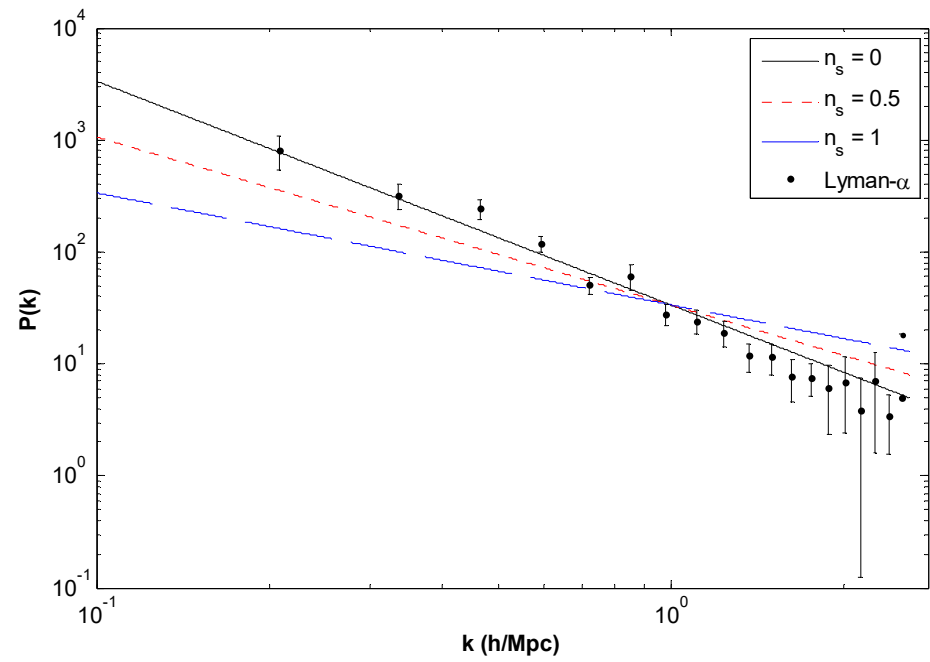

Figure 1. Matter power spectrum on small scales, for various $n_{s}$ with $Q=0$ and $N_{S}=3 \times 10^{8}$, compared to Lyman- $\alpha$ data from eBOSS DR14 [20] compiled in repository here ${ }^{\dagger}$

\footnotetext{
${ }^{\dagger}$ https://github.com/marius311/mpk_compilation/tree/master/dat/lya
} 


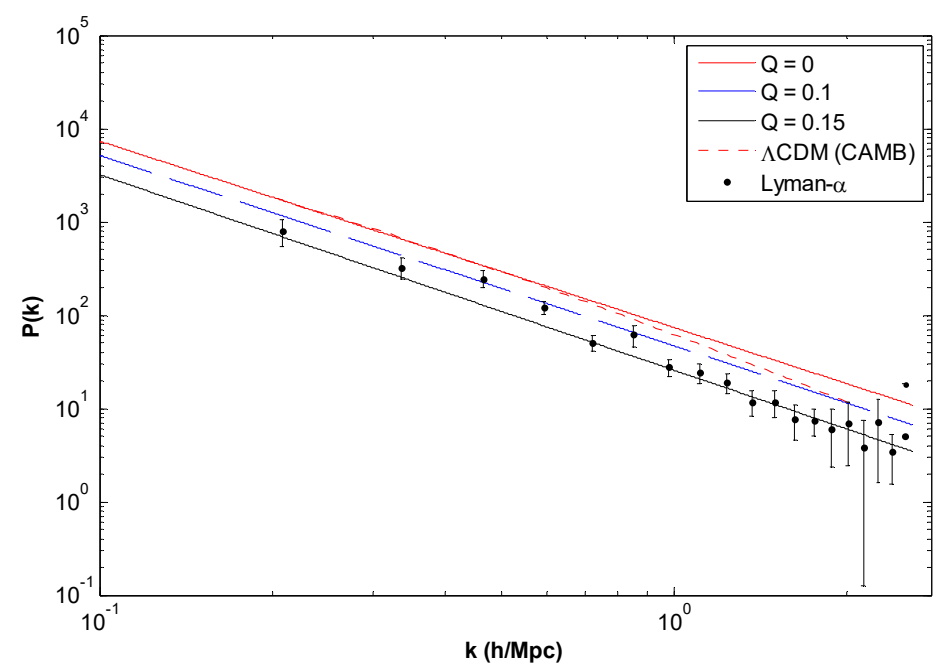

Figure 2. Matter power spectrum on small scales for $n_{s}=0$ with various $Q$ compared to $\Lambda$ CDM model from CAMB and Lyman $-\alpha$ data from [20]

Compared to other models plotted in Figure 1, the power spectrum for $Q=0.15, n_{s}=0$ shown in Figure 2 looks fit better with the data. We also find that, $n_{s}=0$ with non zero coupling is more preferable by data than the invariant-scale $n_{s}=1$. This deviation can be investigated further by numerical study and future data.

\section{Conclusion}

We have investigated matter power spectrum on small scales stimulated by perturbation during matter dominated era. From analytical investigation, we find that the existence of coupling $Q$ between dark matter and scalar field dark energy affects matter power spectrum profile at small scales and shifts the spectrum to the left with decrement. Considering this result as a promising subject to test CSF-DE model, further study needed to anticipate future data.

\section{References}

1. Planck Collaboration, Planck 2018 results. VI. Comological Parameters arXiv:1807.06209v1 (2018)

2. S. Weinberg, The Cosmological Constant Problem Rev. Mod. Phys. 611 (1989)

3. L. Amendola, Phantom Energy Mediates a Long-Range Repulsive Forces Phys. Rev. Lett. 93181102 (2004)

4. L. Amendola, S. Tsujikawa, and M. Sami, Phantom damping of matter perturbation. Phys. Lett. B 632155 (2006)

5. R. R. Caldwell, An Introduction to Quintessence Brazilian Jounal of Physics 302 (2000) 
6. E. J. Copeland, M. Sami, and S. Tsujikawa, Dynamics of Dark Energy Int. J. Mod. Phys. D 151753 (2006)

7. F. A. M. Mulki, and H R T Wulandari, Growth of Matter Perturbation in Quintessence Cosmology American Institute of Physics 1801030002 (2017)

8. B. Ratra, and P. J. E. Peebles, Cosmological consequences of a rolling homogeneous scalar field Phys. Rev. D 373406 (1988)

9. M. Sami, and A. Toporensky, Phantom field and the fate of universe. Mod. Phys. Lett. A 191509 (2004)

10. C. Wetterich, The cosmon model for a asymtotically vanishing time dependent cosmological constant Astron. Astrophys. 301321 (1995)

11. Planck Collaboration, Planck 2015 results. XIV. Dark energy and modified gravity $A \&$ A A 14594 (2016)

12. L. Amendola, Scaling solution in general non-minimal coupling theories Phys. Rev. D 60043501 (1999)

13. L. Amendola, Coupled Quintessence Phys. Rev. D 6204351 (2000)

14. B. Gumjudpai, Naskar, and S. Tsujikawa, Coupled Dark Energy: Towards a general description of the dynamics arXiv:hep-th/0502191v2 (2008)

15. F. A. M. Mulki and H. Wulandari. J. Phys.: Conf. Ser. 1354012007 (2019)

16. V. Pettorino, Coupled and Extended Quintessence: theoretical differences and structure formation arXiv: 0802.1086v2 (2008)

17. F. Piazza, and S. Tsujikawa, JCAP 0407004 (2004)

18. S. Tsujikawa, and M. Sami, A unified approach to scaling solutions in a general cosmological background. Phys. Lett. B 603113 (2004)

19. A. Lewis, A. Challinor, and A. Lasenby, Astrophys. J. 538473 (2000)

20. B. Abolfathi. et al., ApJS, 23542 (2018) 